\title{
Phase synchronization in coupled bistable oscillators
}

\author{
M. R. Jessop $\odot$, W. Li $\odot$, and A. D. Armour $\odot$ \\ Centre for the Mathematics and Theoretical Physics of Quantum Non-Equilibrium Systems and School of Physics and Astronomy, \\ University of Nottingham, Nottingham NG7 2RD, United Kingdom
}

(Received 24 June 2019; accepted 23 January 2020; published 28 February 2020)

\begin{abstract}
We introduce a simple model system to study synchronization theoretically in quantum oscillators that are not simply in limit-cycle states but rather display a more complex bistable dynamics. Our oscillator model is purely dissipative, with a two-photon gain balanced by single- and three-photon loss processes. When the gain rate is low, loss processes dominate and the oscillator has a very low photon occupation number. In contrast, for large gain rates, the oscillator is driven into a limit-cycle state where photon numbers can become large. The bistability emerges between these limiting cases with a region of coexistence of limit-cycle and low-occupation states. Although an individual oscillator has no preferred phase, when two of them are coupled together a relative phase preference is generated which can indicate synchronization of the dynamics. We find that the form and strength of the relative phase preference varies widely depending on the dynamical states of the oscillators. In the limit-cycle regime, the phase distribution is $\pi$ periodic with peaks at 0 and $\pi$, while in the low-occupation regime $\pi$-periodic phase distributions can be produced with peaks at $\pi / 2$ and $3 \pi / 2$. Tuning the coupled system between these two regimes reveals a region where the relative phase distribution has $\pi / 2$ periodicity.
\end{abstract}

DOI: 10.1103/PhysRevResearch.2.013233

\section{INTRODUCTION}

The past few years has seen rapid progress in engineering and probing the properties of nonlinear oscillators in the quantum regime [1-4]. This has stimulated renewed theoretical interest in the properties of such systems, both at the level of individual oscillators and for more complex many-body realizations [5-8]. Of particular interest are phenomena, such as synchronization, which result from an interplay between nonlinearity and nonequilibrium features arising from a combination of gain and loss processes. In the classical regime, the standard paradigm for synchronization involves a nonlinear oscillator in a limit-cycle state which has a well-defined amplitude, but no preferred phase [9,10]. Such oscillators have a tendency to adjust their rhythm to match either a weak external drive or that of other oscillators to which they may be coupled, typically leading to the emergence of a definite phase (or relative phase) for the oscillations. Although synchronization has been studied for a very long time in classical oscillators [9], the systematic study of this behavior in quantum oscillators outside the regime where semiclassical approximations work well [11] is quite recent [12-17].

Studies of synchronization in quantum oscillators have explored issues such as the variety of ways in which the behavior differs from what is found in the semiclassical limit $[16,18-20]$, how best to quantify the degree of synchroniza-

Published by the American Physical Society under the terms of the Creative Commons Attribution 4.0 International license. Further distribution of this work must maintain attribution to the author(s) and the published article's title, journal citation, and DOI. tion in the quantum regime $[15,16,21,22]$, and the relationship between synchronization and entanglement [13,15,19,21,2326]. Much of the work on quantum synchronization has involved simple models such as the quantum van der Pol (QvdP) oscillator [16-18,20] and spin-1 particles [26-28], although a range of other systems have also been considered including atomic ensembles [29,30] and optomechanical systems $[14,15,31]$. Significant efforts have also been devoted to proposing ways in which the behavior could be probed in experiment using systems such as trapped ions [16,22] or superconducting circuits [32].

Although studies of synchronization in the quantum regime have employed a wide range of model systems, they have focused (with occasional exceptions [33]) on systems whose dynamics is essentially just a limit cycle. Here we instead explore how weak coupling generates synchronization, in the form of particular phase preferences, in a quantum oscillator which has a more complex bistable dynamics. We do this by proposing a minimal model for a quantum oscillator that displays a limit-cycle state as well as a low occupationnumber state (in which the oscillator simply fluctuates about the origin) and can be tuned to a bistable regime in which both of these states coexist.

Our model involves only dissipative processes in which photons (quanta) are lost or gained, ensuring that an isolated oscillator never has a preferred phase. The key ingredient of the model responsible for generating bistability is a twophoton gain process. This is balanced by two channels of photon loss in which either a single photon or three photons are annihilated in the oscillator. Different dynamical states of the oscillator (low occupation-number regime, limit cycle, and bistability) are achieved by tuning the relative sizes of the gain and loss rates. In many ways, the model is a logical extension of the much-studied QvdP oscillator which combines 
one-photon gain and two-photon loss [16]. However, the QvdP model always displays a limit cycle, albeit one whose size depends on the ratio of loss and gain rates.

We investigate in detail the phase synchronization that occurs when two of the bistable model oscillators are coupled via a weak photon exchange process. This leads to a rich range of behavior in the relative phase distribution, with a different pattern of phase preferences emerging depending on the underlying dynamical states of the oscillators. When the gain is strong and the oscillators are in limit-cycle states, maximal values of the relative phase distribution form at 0 and $\pi$, matching what is usually found for such states (as seen, e.g., in the QvdP model [16]). For oscillators with low occupation numbers and no limit cycle, however, relative phases of $\pi / 2$ and $3 \pi / 2$ are preferred instead, a result which we argue can be understood as a result of the two-photon gain in this system. All four peaks emerge simultaneously for a small, intermediate parameter regime. Furthermore, this $\pi / 2$-periodic behavior is strongest when the bistability is most pronounced. Interestingly, and in strong contrast to the $\mathrm{QvdP}$ oscillator, we find that the strength of the synchronization in the limit-cycle state does not increase with increasing photon numbers.

The outline of the rest of this paper is as follows. In Sec. II, we start by introducing our bistable quantum oscillator model and exploring its steady state and dynamical properties. Then we investigate the behavior of two coherently coupled oscillators in Sec. III, focusing in particular on the way in which the pattern and strength of features in the relative phase distribution of the system depend on the underlying dynamical properties of each oscillator. We conclude in Sec. IV and the Appendixes provide details about aspects of the calculations employed.

\section{BISTABLE OSCILLATOR SYSTEM}

\section{A. 321-oscillator model}

Our oscillator model involves three dissipative processes, as illustrated in Fig. 1(a). A two-photon gain process with rate $\kappa_{2}$ drives the oscillator to higher photon numbers, while a one-photon loss process damps it at a rate $\kappa_{1}$; an additional three-photon loss process at rate $\kappa_{3}$ is included to stabilize the system, ensuring that it has a steady state for any strength of the gain. The master equation for a single oscillator in the interaction picture is given by $[16,34,35]$

$$
\dot{\rho}=\mathcal{L} \rho=\kappa_{1} \mathcal{D}[\hat{a}](\rho)+\kappa_{2} \mathcal{D}\left[\left(\hat{a}^{\dagger}\right)^{2}\right](\rho)+\kappa_{3} \mathcal{D}\left[\hat{a}^{3}\right](\rho),
$$

where $\hat{a}$ is the oscillator lowering operator and we have defined $\mathcal{D}[\hat{C}](\rho)=\hat{C} \rho \hat{C}^{\dagger}-\frac{1}{2}\left\{\hat{C}^{\dagger} \hat{C}, \rho\right\}$. Our model makes an interesting contrast with the QvdP oscillator [16,17], where one-photon gain is balanced by two-photon loss. The presence of a nonlinear gain process in our model leads to important features, such as bistability, not seen for the QvdP.

The steady-state properties are readily found by exploiting the fact that the system is purely dissipative, so that the dynamics of the diagonal and off-diagonal matrix elements of the density operator in the number (Fock) basis are decoupled $[36,37]$. The master equation can therefore be rewritten as a
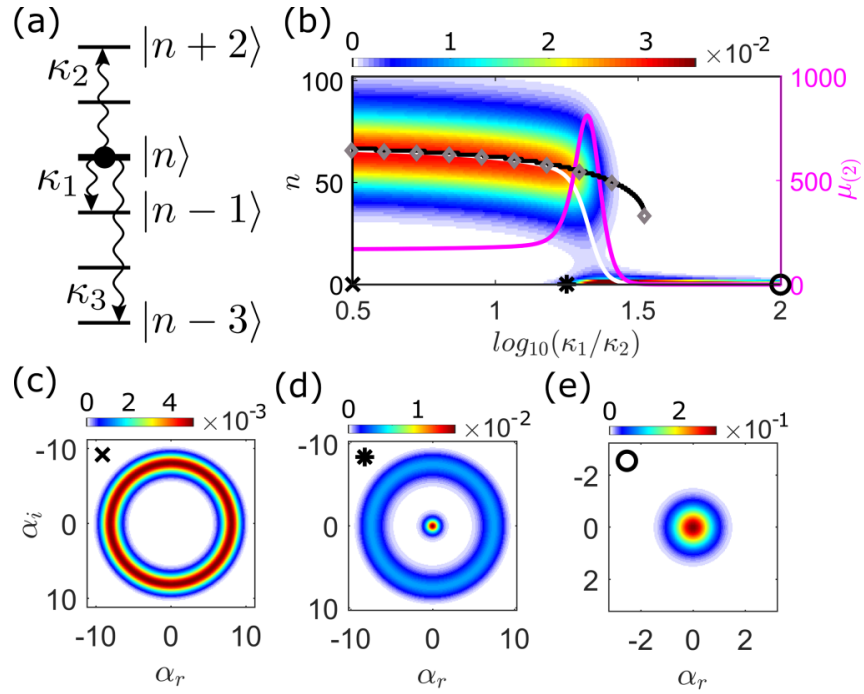

(e)

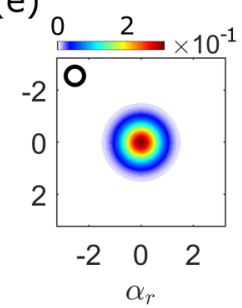

FIG. 1. (a) The three dissipative processes of the oscillator: two-photon gain, single-photon loss, and three-photon loss, at rates $\kappa_{2}, \kappa_{1}$, and $\kappa_{3}$ respectively. (b) Steady-state properties as a function of $\kappa_{1} / \kappa_{2}$ for fixed $\kappa_{3}=\kappa_{2} \times 10^{-2}$. A color scale shows the photonnumber distribution $P_{n}$ (for $n>0$ ), with the average photon number $\langle n\rangle$ calculated numerically (white line), location of the peak in $P_{n}$ away from $n=0$ where it exists (solid black line), and the mean-field prediction $n_{+}$(gray diamonds), superposed. Large photon number states are occupied when the gain is sufficiently large (black cross), the fixed point state is predominantly occupied if the loss dominates (black circle), and a bimodal distribution appears in an intermediate region (black star). Also shown is the second moment $\mu_{(2)}$ (solid magenta line). The corresponding Wigner functions, $W\left(\alpha_{r}, \alpha_{i}\right)$, are for (c) limit-cycle $\left(\kappa_{1} / \kappa_{2}=10^{0.5}\right)$, (d) bistability $\left(\kappa_{1} / \kappa_{2}=10^{1.25}\right)$ and (e) fixed point $\left(\kappa_{1} / \kappa_{2}=10^{2}\right)$.

set of $k$ equations

$$
\dot{\rho}^{(k)}=\mathcal{M}^{(k)} \rho^{(k)},
$$

where $\rho_{n}^{(k)}=\langle n|\rho| n+k\rangle$, with $|n\rangle$ being the $n$th number state and $\mathcal{M}^{(k)}$ being a matrix. For the diagonal elements, writing out Eq. (1) explicitly leads to the coupled set of equations

$$
\dot{P}_{n}=-G_{n} P_{n}+A_{n+1} P_{n+1}+B_{n-2} P_{n-2}+C_{n+3} P_{n+3},
$$

for the probabilities, $P_{n}=\langle n|\rho| n\rangle$, with

$$
\begin{aligned}
G_{n} & =A_{n}+B_{n}+C_{n}, \\
A_{n} & =\kappa_{1} n, \\
B_{n} & =\kappa_{2}(n+2)(n+1), \\
C_{n} & =\kappa_{3}(n-2)(n-1) n,
\end{aligned}
$$

from which the form of $\mathcal{M}^{(0)}$ follows. In the steady state, the off-diagonal terms $\rho^{(k \neq 0)}$ all go to zero and the eigenvector of $\mathcal{M}^{(0)}$ with zero eigenvalue gives the $P_{n}$ distribution.

\section{B. Steady-state properties}

The steady state of the oscillator can be characterized by the behavior of the $P_{n}$ distribution along with the Wigner distribution [35], $W\left(\alpha_{r}, \alpha_{i}\right)$. Figures 1(b)-1(e) show how the state of the system evolves as the ratio $\kappa_{1} / \kappa_{2}$ is changed for a small (fixed) value of $\kappa_{3} / \kappa_{2}$. When the nonlinear gain, $\kappa_{2}$, 
is sufficiently large, the oscillator is driven to large photon numbers with an almost Gaussian $P_{n}$ distribution centered at a value $\langle n\rangle=\sum_{n} n P_{n} \gg 1$ [see Fig. 1(b)]. The corresponding Wigner distribution possesses a ring of maxima [Fig. 1(c)]; we classify this as a limit-cycle (LC) state, as it has a well-defined average amplitude, but no preferred phase [16]. In the opposite limit of dominant loss $\left(\kappa_{1} / \kappa_{2} \gg 1\right)$, the oscillator is damped to the lowest photon number states, leading to a sharp peak in the $P_{n}$ distribution at $n=0$. In this regime, the Wigner distribution displays a single maximum at the origin [Fig. 1(e)] and we call this a fixed point (FP) state. In between these limits, we find bistability (B) where features from both the LC and FP states can be found in the Wigner distribution [see Fig. 1(d)] and two peaks of similar area feature in the $P_{n}$ distribution [38].

The bimodality of the $P_{n}$ distribution is captured by a sharp peak in the second moment [39] $\mu_{(2)}=\left\langle n^{2}\right\rangle-\langle n\rangle^{2}$, as shown in Fig. 1(b). The distributions with the highest values of $\mu_{(2)}$ are found to be those with the most pronounced bistability, i.e., with two peaks of comparable area that are separated by a significant gap.

We use a standard mean-field (or semiclassical) approach to understand the origin of the oscillator's dynamical states (the details are described in Appendix A). Two physically relevant mean-field solutions for the average photon number are found: a zero photon solution $n_{0}=0$ (corresponding to the fixed point) and a nonzero solution $n_{+}=\left[\kappa_{2}+\sqrt{\kappa_{2}^{2}-3 \kappa_{1} \kappa_{3}}\right] /\left(3 \kappa_{3}\right)$ (corresponding to the limit cycle). Linear stability analysis reveals that the zero photon solution $n_{0}$ is always stable. The nonzero solution $n_{+}$, however, is only real and stable for the parameters

$$
3 \kappa_{1} \kappa_{3}<\kappa_{2}^{2} .
$$

As such, the mean-field approach predicts a region of bistability associated with the coexistence of two stable solutions in the parameter regime defined by Eq. (4).

These predictions are compared with the behavior of the $P_{n}$ distribution in Fig. 1(b). The $n_{+}$solution (gray diamonds) agrees remarkably well with the location of the peak away from $n=0$ that develops in the $P_{n}$ distribution (solid black line).

The behavior of the oscillator as a function of both $\kappa_{1} / \kappa_{2}$ and $\kappa_{3} / \kappa_{2}$ is summarized in Fig. 2. A classification of the oscillator state as either FP, B, or LC, based on the corresponding Wigner function (see Appendix B for details) is shown together with $\mu_{(2)}$ in Fig. 2(a), while the behavior of the average occupation number is shown in Fig. 2(b).

The boundary between the FP and B regions is well described by the mean-field prediction [Eq. (4)] over a wide range of parameters, although it does eventually fail when photon numbers become sufficiently small. However, there is no sharp transition between the LC and B regions, but rather a gradual crossover reflecting a very gradual change between the states. Hence, the boundary between these two regions falls in different places according to the details of how we choose to define it. Two different definitions are illustrated in Fig. 2: As the line where the peak at the origin of the Wigner function disappears entirely and a less conservative choice which seeks to delimit where the peak at the origin becomes extremely small (although it does still exist), dropping below a

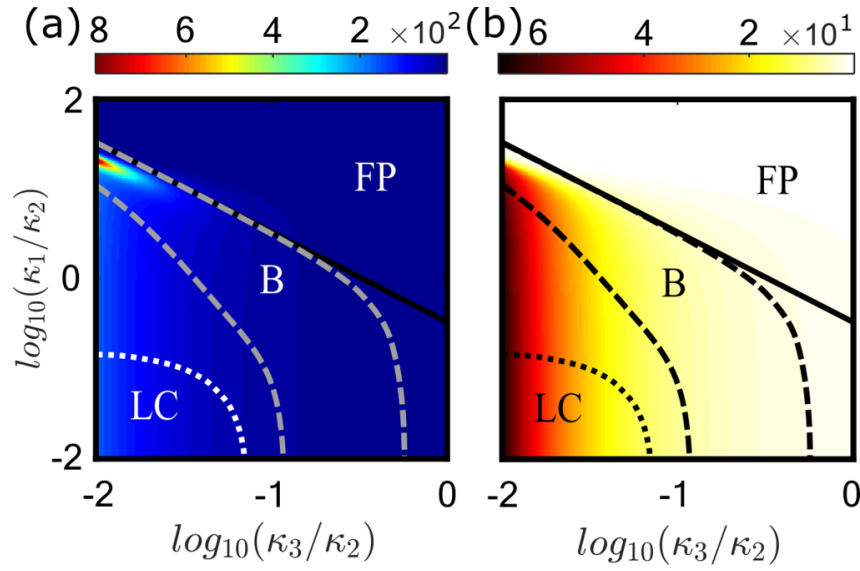

FIG. 2. Steady-state behavior of (a) the second moment $\mu_{(2)}$ and (b) average occupation number $\langle n\rangle$ overlaid with boundaries between the fixed point (FP), limit-cycle (LC), and bistable (B) regimes obtained by analyzing the peaks in the radial Wigner distribution $W(r=|\alpha|)$ (see Appendix B for details). The FP-B boundary (upper dashed curve) agrees well with the appearance of the stable nonzero mean field solution, $n_{+}$[Eq. (4)] (full line). The LC-B boundary is shown using two different approaches (see main text for details): The dotted line indicates where the peak at the origin of the Wigner function disappears entirely, while the (lower) dashed line indicates the edge of a bistable region in which the Wigner peak at the origin remains non-negligible in size. The second moment is maximal within the bistable region where the corresponding LC contains a large occupation number (i.e $\kappa_{2} \gg \kappa_{3}$ ); elsewhere, it is rather smooth. The average photon number distribution $\langle n\rangle$ is largest deep within the LC regime (red) and lowest for the fixed point regions (white), but does not provide any direct indication of bistability.

threshold value (defined as a difference between the height of the Wigner function for the local maximum at the origin and that at the nearby local minimum of $10^{-4}$, see Appendix B). The latter boundary, although necessarily somewhat arbitrary, is perhaps more appropriate as it leads to a much narrower bistable region at small $\kappa_{3} / \kappa_{2}$, which accords with what we would expect from the existence of a sharp peak in $\mu_{(2)}$ and the corresponding relatively rapid change between low- and high- $\langle n\rangle$ regions that occurs in this regime [see Fig. 2(b)].

Average photon numbers tend to decrease overall as $\kappa_{3} / \kappa_{2}$ is increased and, although we can still use the Wigner distribution to categorize the oscillator states, the amplitude of the limit-cycle states get smaller, necessarily reducing the distinction between limit-cycle and fixed point states (as the corresponding peaks increasingly overlap). While it should be possible, at least in principal, to reconstruct the Wigner function of the oscillator from measurements, it would obviously be much easier to identify the boundaries using the clear signatures that arise for small $\kappa_{3} / \kappa_{2}$ values (such as the the peak in $\mu_{(2)}$ or even the rapid change that occurs in $\langle n\rangle)$.

\section{Dynamical properties}

We now turn to the dynamical properties of the system to understand whether the bistable states we have identified are also metastable in the sense that they display slow switching 

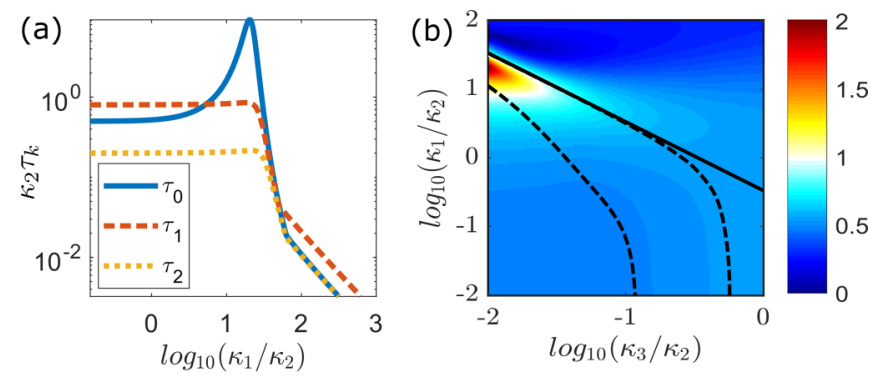

FIG. 3. (a) The slowest timescales $\tau_{k}$ of the oscillator for $k=$ $0,1,2$ with $\kappa_{3}=\kappa_{2} \times 10^{-2}$ and (b) the metastability $M$ [Eq. (5)] plotted on a logarithmic scale. The phase boundaries obtained using Wigner functions (dashed lines) and the mean field calculation (full line) are shown.

between the two states. To investigate this, we start by calculating how the eigenvalues with largest real parts (i.e., least negative, but nonzero) of the system behave [40-42].

The largest eigenvalue of the matrix $\mathcal{M}^{(k)}$ in Eq. (2), $\lambda_{1}^{(k)}$, can be used to obtain the slowest timescale associated with the dynamics of $\rho^{(k)}, \tau_{k}=-1 / \operatorname{Re}\left(\lambda_{1}^{(k)}\right)$, examples of which are shown in Fig. 3(a). The $k=0$ case, $\tau_{0}$, describes the relaxation of the diagonal elements and becomes very large for a range of $\kappa_{1} / \kappa_{2}$. The other timescales $\tau_{1,2}$, describe the relaxation of phase preferences in the system. Although they never become as large as the peak values of $\tau_{0}$ and display no obvious signature of the bistability, they do change significantly as the system evolves from FP to LC states, becoming orders of magnitude larger in the latter case.

In this system, the emergence of a single very slow timescale signals metastability [42]. The resulting separation of timescales can be quantified through the ratio of the largest (least negative) eigenvalues,

$$
M=\frac{\operatorname{Re}\left(\lambda_{2}^{(0)}\right)}{\operatorname{Re}\left(\lambda_{1}^{(0)}\right)} .
$$

$M$ is much larger than unity when there is a wide separation of timescales. The behavior of $M$ as a function of $\kappa_{3} / \kappa_{2}$ and $\kappa_{1} / \kappa_{2}$ is shown in Fig. 3(b); note that the color scale is white where $M=10$ which we take as a rough (if somewhat arbitrary) threshold for metastability. The parameter range identified as metastable by this criterion is, unsurprisingly, much smaller than that labeled on the basis of the steady state as bistable in Fig. 2(a). Nevertheless, the peaks in $M$ and the second moment plotted in Fig. 2(a) match up relatively well.

We can get more insight into the dynamical properties of the system by looking at quantum jump trajectories [43], obtained by unravelling the master equation. The system is evolved in time with the non-Hermitian Hamiltonian $H_{M C}=-\frac{i}{2}\left(\kappa_{1} \hat{a}^{\dagger} \hat{a}+\kappa_{2} \hat{a}^{2}\left(\hat{a}^{\dagger}\right)^{2}+\kappa_{3}\left(\hat{a}^{\dagger}\right)^{3} \hat{a}^{3}\right)$. For each short time step, quantum jumps of three different kinds (one-photon loss, two-photon gain, or three-photon loss process) can occur with a probability that depends on the state of the system (e.g., the probability of two photon gain occurring over the interval $\delta t$ is given by $\kappa_{2}\left\langle\psi(t)\left|\hat{a}^{2}\left(\hat{a}^{\dagger}\right)^{2}\right| \psi(t)\right\rangle \delta t$, where $|\psi(t)\rangle$ is the state of the oscillator).

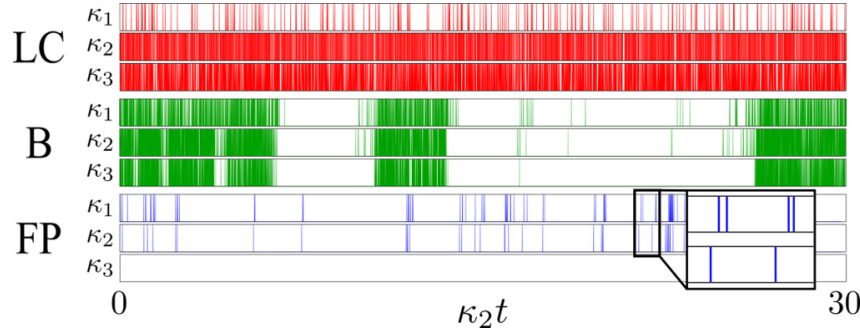

FIG. 4. Sample quantum trajectories for each of the oscillator states illustrating the frequency of the different jump processes for (top) limit-cycle $\left(\kappa_{1}=\kappa_{2} \times 10^{1 / 2}\right)$, (middle) bistable $\left(\kappa_{1}=\kappa_{2} \times\right.$ $\left.10^{5 / 4}\right)$, and (bottom) fixed-point $\left(\kappa_{1}=\kappa_{2} \times 10^{7 / 4}\right)$ states (with $\kappa_{3}=$ $\kappa_{2} \times 10^{-7 / 4}$ throughout). The individual jump processes involving one-photon loss (rate $\kappa_{1}$ ), two-photon gain (rate $\kappa_{2}$ ), and three-photon loss $\left(\right.$ rate $\kappa_{3}$ ) are indicated. The bistable oscillator can be seen to flip intermittently between LC-like and FP-like behavior. In the FP state, the one-photon loss jumps occur in pairs soon after each two-photon gain jump (see magnified portion of the lower panel).

The frequency of the different jump processes is illustrated in Fig. 4 for sample trajectories obtained for parameters corresponding to the three different states of the system (LC, FP, and B). Within the LC state, the oscillator displays a high level of activity (i.e., frequent jumps); in this case, the large photons numbers involved mean that the two- and three-photon processes dominate over the single-photon one. In contrast, all jump processes are strongly suppressed (and the three-photon loss especially so) in the FP state because of the very low occupation numbers in this regime. Within the region which is both bistable and metastable (based on the behavior of the eigenvalues), the oscillator switches between periods in which it exhibits high and low levels of activity; the switching continues indefinitely, never settling into one state or the other. This intermittency in the dynamics of the trajectories is consistent with our interpretation of this regime as metastable [42].

\section{SYNCHRONIZATION OF COUPLED OSCILLATORS}

We now explore how phase ordering and synchronization occurs when two of these oscillators are coupled together weakly. For simplicity, we consider two identical oscillators and assume a coherent (photon-exchange) interaction of the form $[16,17,22]$

$$
\hat{H}_{J}=\hbar J\left(\hat{a}_{1}^{\dagger} \hat{a}_{2}+\hat{a}_{1} \hat{a}_{2}^{\dagger}\right)
$$

where $\hat{a}_{j}$ is a lowering operator for oscillator $j$ and $J$ is the strength of the coupling. The master equation of the coupled system is given by

$$
\dot{\rho}=-\frac{i}{\hbar}\left[\hat{H}_{J}, \rho\right]+\sum_{j=1,2} \mathcal{L}_{j} \rho,
$$

where the dissipation terms follow from Eq. (1): $\mathcal{L}_{j} \rho=$ $\kappa_{1} \mathcal{D}\left[\hat{a}_{j}\right](\rho)+\kappa_{2} \mathcal{D}\left[\left(\hat{a}_{j}^{\dagger}\right)^{2}\right](\rho)+\kappa_{3} \mathcal{D}\left[\hat{a}_{j}^{3}\right](\rho)$.

Classical limit-cycle oscillators typically synchronize when they are coupled together weakly, developing a preference for one or more relative phase values $[9,44]$. In the quantum regime, the phase states $|\varphi\rangle=\sum_{n=0}^{\infty} \mathrm{e}^{i n \varphi}|n\rangle$ can 

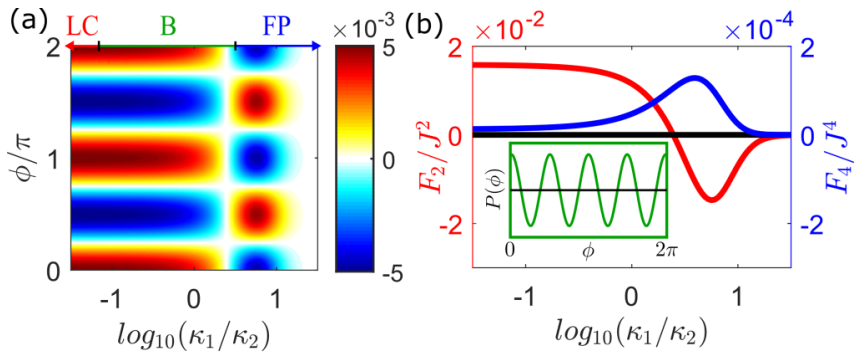

FIG. 5. (a) $[P(\phi)-1 / 2 \pi]\left(\kappa_{2}^{2} / J^{2}\right)$ as a function of $\kappa_{1} / \kappa_{2}$ (calculated with perturbation theory), with $J / \kappa_{2}=10^{-2}$ and $\kappa_{3}=\kappa_{2} \times$ $10^{-1}$. The range of $\kappa_{1} / \kappa_{2}$ shown spans the three states (FP, B, and LC). Very weak single-photon loss $\left(\kappa_{1} \ll \kappa_{2}\right)$ leads to coupled limit-cycles with peaks at 0 and $\pi$. As the single-photon loss rate is increased, this $\pi$-periodic pattern vanishes before then reappearing with peaks at $\pi / 2$ and $3 \pi / 2$. Eventually, very strong singlephoton loss $\left(\kappa_{1} \gg \kappa_{2}\right)$ again suppresses the pattern. (b) The dominant Fourier coefficients $F_{2}$ (red) and $F_{4}$ (blue), defined in the main text (here units are chosen such that $\kappa_{2}=1$ ). $F_{2}$ accounts for the $\pi$-periodic component of the relative phase distribution and its sign determines the position of the peaks; $F_{4}$ is the next largest, though it is much less important than $F_{2}$, except for the region in which $F_{2}$ passes through zero. The corresponding $\frac{\pi}{2}$-periodic $P(\phi)$ distribution for the case where $F_{2}$ is zero is shown in the inset; the peak-to-peak height is $10^{-4}$ in units of $\left(J / \kappa_{2}\right)^{4}$.

be used to construct a relative phase probability distribution $[19,20,22,45-47]$

$$
\begin{aligned}
P(\phi) & =\frac{1}{2 \pi} \sum_{n, m=0}^{\infty} \sum_{k=\max (n, m)}^{\infty} e^{i \phi(m-n)}\langle n, k-n|\rho| m, k-m\rangle, \\
& =\frac{1}{2 \pi}+\frac{1}{\pi} \operatorname{Re}\left[\sum_{k=1}^{\infty} e^{i k \phi} \sum_{n, m=0}^{\infty}\langle n+k, m|\rho| n, m+k\rangle\right],
\end{aligned}
$$

where $\phi=\varphi_{1}-\varphi_{2}$ is the relative phase of the two oscillators. If the two oscillators are uncoupled, their phases are independent and the relative phase distribution is uniform, $P(\phi)=$ $\frac{1}{2 \pi}$. In the following, we use the relative phase distribution to explore the impact of coupling on the behavior of our oscillator model in each of the dynamical states it displays.

Since we are interested in the effect of a very weak coupling between the oscillators, we use a perturbation method $[18,19,28]$ to calculate the relative phase distribution, details of which are described in Appendix C. Figure 5(a) shows how the relative phase distribution evolves as a function of $\kappa_{1} / \kappa_{2}$ and the system passes from FP to LC via the bistable region. Interestingly, the system displays $\pi$-periodic phase distributions with peaks which reach a similar size in both the $\mathrm{LC}$ and FP regimes. However, the location of the peaks are different in the two cases and in between (the bistable region) the phase distribution appears to flatten.

A more detailed picture of the phase behavior is obtained by looking at the Fourier coefficients of the relative phase distribution, $F_{k}=\operatorname{Re}\left[\sum_{n, m=0}^{\infty}\langle n+k, m|\rho| n, m+k\rangle\right]$. For our coupled oscillator system, these coefficients are only nonzero for even $k$ and typically get smaller very rapidly with increasing $k$. The two most important coefficients $F_{2}$ and $F_{4}$ are shown in Fig. 5(b) (calculated to second and fourth order in $J$ respectively). The $\pi$ periodicity of the distribution apparent in Fig. 5(a) stems from the fact that the magnitude of $F_{2}$ is almost everywhere much larger than that of $F_{4}$. However, as the system changes from LC to FP (via the bistablity) $F_{2}$ changes sign to produce the shift in the locations of the peaks; as $F_{2}$ passes through zero $F_{4}$ dominates, giving rise to an unusual $\pi / 2$ periodic distribution.

The different $\pi$-periodic patterns that arise in the phase distribution can be understood using simple arguments that exploit the specific characteristics of the system in the LC and FP states. Well within the LC regime, photon occupation numbers are large and semiclassical approaches work well, as Fig. 1(b) illustrates for the single oscillator case. A straightforward calculation described in Appendix D recovers the preference for relative phases of 0 and $\pi$ which is generic for coherently coupled limit-cycle oscillators $[16,19]$.

The pattern of peaks in the relative phase distribution at $\pi / 2$ and $3 \pi / 2$ seen for the FP can be understood by focusing on the limit where $\kappa_{1} / \kappa_{2} \gg 1$. In this regime, photon occupation numbers are very small, the value of $\kappa_{3}$ becomes irrelevant, and we can simplify the perturbation theory calculation by assuming only the three lowest Fock states of the oscillators have non-negligible occupations (see Appendix C for details). In this limit, we find

$$
P(\phi)=\frac{1}{2 \pi}+J^{2} f\left(\kappa_{1}, \kappa_{2}\right)\left[P_{1}^{2}-P_{0} P_{2}\right] \cos (2 \phi),
$$

where $f\left(\kappa_{1}, \kappa_{2}\right)$ is a (positive-valued) function of the two rates, while $P_{0}, P_{1}$, and $P_{2}$ are the occupation probabilities of the three lowest Fock states for the corresponding uncoupled oscillator. Clearly the behavior is always $\pi$ periodic, but the positions of the maxima depend on the occupation probabilities which in turn depend on the details of how the system is driven and damped in this regime. In our oscillator model, the two-photon driving gives a boost to $P_{2}$, generating a steady state with a photon distribution where $P_{2} P_{0} / P_{1}^{2}>1$, which leads to the peaks at $\pi / 2$ and $3 \pi / 2$ seen in Fig. 5. Thus, the fact that our oscillator model displays a $\pi$-periodic relative phase distribution with peaks at $\pi / 2$ and $3 \pi / 2$ is most likely due to the particular form of the lowest order nonlinearity in the system, two-photon gain, which shapes the steady-state number distribution.

We can test this idea further by applying a very similar analysis to coherently coupled oscillators with different gain and loss processes (within the same low-occupation number limit). In particular, for an oscillator coupled to a thermal bath (with one-photon gain and loss related by a ratio of rates $\bar{n} /(\bar{n}+1)$, with thermal occupation number $\bar{n})$ and the QvdP oscillator [16] (one-photon gain and two-photon loss), one obtains expressions like that in Eq. (10). In each case, $f$ takes a form that is different in detail, but the sign of $P_{1}^{2}-P_{0} P_{2}$ still determines the location of the peaks. For the QvdP oscillator in the strongly damped limit $P_{2} P_{0}<P_{1}^{2}$, and hence one finds the same phase behavior as in the large photon number regime [16] (peaks at 0 and $\pi$ ). In contrast, for a thermal oscillator $P_{2} P_{0}=P_{1}^{2}$ and so no phase preference is expected. This latter case also involves a fixed point state for the oscillator which allows us to exclude the possibility that 


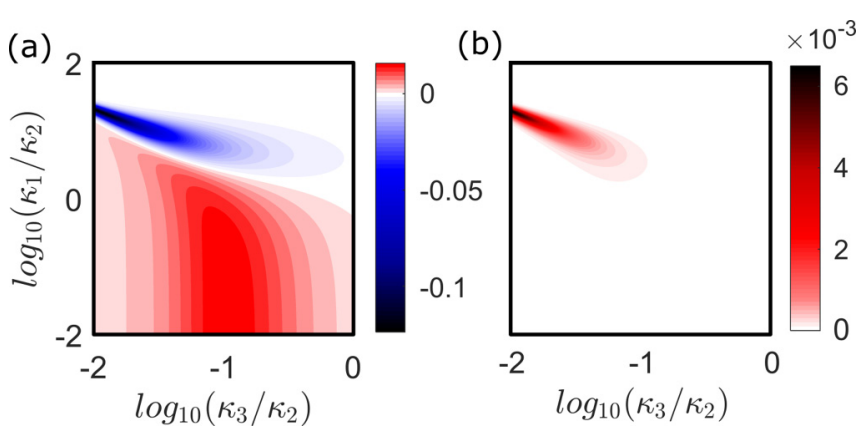

FIG. 6. Behavior of (a) $F_{2} / J^{2}$ and (b) $F_{4} / J^{4}$, the two most dominant Fourier coefficients of the relative phase distribution scaled with coupling strength as a function of the relative loss-gain rates (in units such that $\kappa_{2}=1$ ).

the phase pattern we see in our system at low photon numbers is simply due to the underlying fixed point state.

Finally, Fig. 6 shows how the components $F_{2}$ and $F_{4}$ behave for a range of $\kappa_{3} / \kappa_{2}$ as well as $\kappa_{1} / \kappa_{2}$. The change between negative and positive values of $F_{2}$ is sharpest, and the corresponding peak in $F_{4}$ strongest, in the region where $\kappa_{3} / \kappa_{2} \ll 1$, which is where the system also displays metastability.

Figure 6 also reveals a somewhat surprising feature of the phase behavior. The strongest phase preferences are not associated with the limit-cycle regime. Furthermore, deep within the limit-cycle regime, the magnitude of $F_{2}$ starts to saturate, even as the average photon number continues to grow, i.e., as $\kappa_{3} / \kappa_{2}$ is further reduced in the bottom left quadrant of Fig. 6(a). This is in contrast to other quantum limit-cycle oscillators, such as the QvdP oscillator, for which phase synchronization effects are enhanced by increasing the photon number [16]. We can gain some insight into why this occurs from the phase dynamics of an uncoupled oscillator. At least in the semiclassical regime of large photon numbers, we expect to see stronger synchronization effects emerge when coupling is introduced in systems where the phase diffusion is weaker $[9,19]$.

Using an approximate analytic approach (see Appendix E for details), we find that the phase diffusion rate is simply proportional to $\kappa_{2}$ in the regime where gain dominates over losses and photon numbers are large. This contrasts with the behavior of the QvdP oscillator where the phase diffusion is $\propto 1 /\langle n\rangle$, which is why for this system synchronization effects get stronger as photon numbers are increased.

\section{CONCLUSIONS}

We have introduced a simple oscillator model with a twophoton gain process balanced by one- and three-photon losses that can be used to engineer a bistable oscillator state. The bistability occurs when the gain-loss rates are tuned between regimes where the oscillator displays a limit-cycle state characterized by a large amplitude (but no preferred phase) and a fixed-point state where occupation numbers are low. Quantum trajectory simulations show clear evidence of metastability in the bistable region, signalled by intermittency in the frequency of the quantum jumps.
When two such oscillators are (coherently) coupled together weakly, their relative phase distribution displays a rich pattern of behavior. While the system has the usual predominantly $\pi$-periodic distribution with peaks at 0 and $\pi$ within the limit-cycle regime, in the limit of low occupation numbers the peaks appear at $\pi / 2$ and $3 \pi / 2$. In between these two regimes, where the bistability arises, the distribution can instead be $\pi / 2$ periodic.

In the limit-cycle regime where photon numbers tend to be large, the form of the relative phase distribution can be understood from mean-field models which show that the preferences are determined by the form of the coupling between the oscillators, as is the case in the classical regime. In contrast, for very low photon occupation numbers, our analysis shows instead that for the coherent coupling we consider the behavior is determined by the precise form of the (nonlinear) gain-loss terms, which in turn determine the number distribution. For the case of the 321 oscillator at very low occupation numbers, the two-photon driving leads to a number distribution for the oscillators which gives rise to the preferred phases of $\pi / 2$ and $3 \pi / 2$, something which is not seen for either thermally distributed oscillators or the QvdP model.

Our goal in this work has been to explore the properties of the relative phase distribution and its connection to the underlying oscillator dynamics in the simplest possible model system displaying limit cycles and bistability. We plan to address the question of how the complex behavior we uncovered could be realized, and detected, in future work. It would also be interesting to investigate how the dynamics is affected by strong couplings [48] and the patterns of phase behavior that arise in systems where more than two oscillators are coupled together $[14,16,49]$. Beyond synchronization effects, there are other aspects of the model system that we have introduced here that will be worth studying in the future. For example, we have not explored the mechanism by which slow switching occurs in the metastable regime. Finally, a comparison of the full quantum dynamics of the system with a semiclassical model including fluctuations, e.g., at the level of a FokkerPlanck equation for the Wigner function, would be worth carrying out to uncover which features of the behavior can be attributed to the quantum character of the fluctuations in the system $[16,50]$.

\section{ACKNOWLEDGMENTS}

This work was supported by the Engineering and Physical Sciences Research Council (Grant No. EP/N50970X/1) through a studentship held by M.R.J. W.L. acknowledges support from the UKIERI-UGC Thematic Partnership No. IND/CONT/G/16-17/73 and EPSRC Grants No. EP/M014266/1 and No. EP/R04340X/1.

\section{APPENDIX A: MEAN-FIELD ANALYSIS}

An equation of motion for the expectation value of the annihilation operator, $\frac{d}{d t}\langle\hat{a}\rangle=\operatorname{Tr}[\hat{a} \dot{\rho}]$, is found from the master equation [Eq. (1)]:

$$
\frac{d}{d t}\langle\hat{a}\rangle=-\frac{\kappa_{1}}{2}\langle\hat{a}\rangle+\kappa_{2}\left\langle\hat{a}^{2} \hat{a}^{\dagger}\right\rangle-\frac{3 \kappa_{3}}{2}\left\langle\left(\hat{a}^{\dagger}\right)^{2} \hat{a}^{3}\right\rangle .
$$


In a mean-field (or semiclassical) approach, we break the correlations between operators such that $\langle\hat{A} \hat{B}\rangle \rightarrow\langle\hat{A}\rangle\langle\hat{B}\rangle$. We choose to carry out this approximation without splitting the expectation values of operators, e.g., $\left\langle\hat{a}^{2} \hat{a}^{\dagger}\right\rangle$ is not normal ordered (in which case it would become $2\langle\hat{a}\rangle+\left\langle\hat{a}^{\dagger} \hat{a}^{2}\right\rangle$ ).

Making the substitution $\langle a\rangle=r \mathrm{e}^{i \varphi}$ where $r$ and $\varphi$ are classical amplitude and phase variables allows us to rewrite Eq. (A1) as

$$
\dot{r}+i r \dot{\varphi}=-\frac{3 \kappa_{3}}{2} r^{5}+\kappa_{2} r^{3}-\frac{\kappa_{1}}{2} r .
$$

Evaluating the real and imaginary parts separately leads to $\dot{\varphi}=0$ and

$$
\dot{r}=-\frac{r}{2}\left[3 \kappa_{3} r^{4}-2 \kappa_{2} r^{2}+\kappa_{1}\right] .
$$

The steady-state solutions (in terms of average photon numbers, $n=r^{2}$ ) are $n_{0}=0$ and

$$
n_{ \pm}=\frac{\kappa_{2}}{3 \kappa_{3}}\left[1 \pm \sqrt{1-\frac{3 \kappa_{1} \kappa_{3}}{\kappa_{2}^{2}}}\right],
$$

which are subject to the constraints of being both positive and real. The stability of the solutions can be determined, e.g., through the properties of the relevant Jacobian.

The negative branch, $n_{-}$, is never both physical and stable; the zero-photon solution, $n_{0}$, is always stable; and the positive branch, $n_{+}$, is stable whenever it is physical, i.e., for $3 \kappa_{1} \kappa_{3}<$ $\kappa_{2}^{2}$. This mean-field calculation results in two stable solutions for the average photon number and therefore a predicted bistability in the photon number for the parameters where $n_{+}$ is stable.

We note that carrying out the mean-field approximation after normally ordering the operators in Eq. (A1) leads to somewhat different results; the two nonzero branches are $n_{ \pm}=\left[\kappa_{2} \pm \sqrt{\kappa_{2}^{2}+3 \kappa_{3}\left(4 \kappa_{2}-\kappa_{1}\right)}\right] /\left(3 \kappa_{3}\right), n_{+}$is stable for $\frac{\kappa_{1}}{\kappa_{2}}<4+\frac{\kappa_{2}}{3 \kappa_{3}}$, and $n_{-}$is never stable. Interestingly, in this approach the fixed point $n_{0}$ becomes unstable for $\kappa_{1}<4 \kappa_{2}$. However, the corresponding mean-field boundary marking the onset of bistability (two stable solutions) matches up with the full quantum calculation less well than the approach without normal ordering, which is why we have chosen to use the latter in the main text. Notice, however, that the difference between the fixed-point and bistability boundaries calculated using the different mean-field equations gets smaller as $\kappa_{3} / \kappa_{2}$ is reduced, which typically corresponds to going to larger average occupation numbers [see Fig. 2(b)].

\section{APPENDIX B: CATEGORIZING STATES}

The boundaries between limit-cycle (LC), fixed point (FP), and bistable (B) regions in Fig. 2 are drawn on the basis of the properties of the corresponding Wigner function for each set of parameters [see Fig. 7(a)]. The most straightforward way of classifying the state of the system is on the basis of whether a maximum exists at the origin [50] together with a local maximum at $r>0$ (B), just a maximum at the origin (FP), or just a maximum at a nonzero radius (LC) [examples of each of these cases are shown in Fig. 7(c)]. To determine whether the Wigner function has a maximum at the origin, we exploit the radial symmetry and calculate it as a function of the radius,
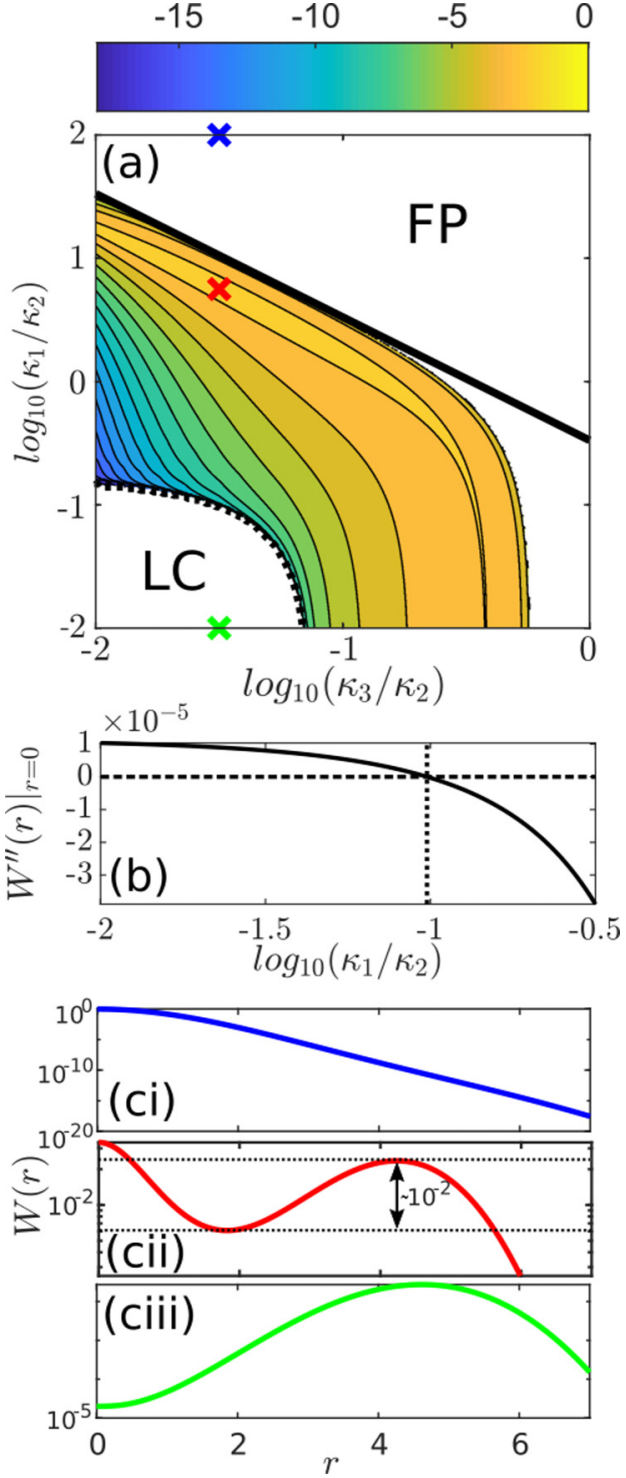

FIG. 7. (a) Mapping of the contrast in the Wigner function across the region of bistability, plotted on a log scale with a series of contours from $10^{-18}$ to $10^{-2}$. The contrast is defined as $\min \left(W_{0}, W_{+}\right)-$ $W_{-}$, where $W_{0}$ and $W_{+}$are the values of $W(r)$ at the two maxima of the bistability at $r=0$ and $r>0$ respectively, and $W_{-}$is the value of $W(r)$ at the local minimum between them. The white regions indicate Wigner functions with only a single maximum, the dashed black line indicates the locus where $\left.W^{\prime \prime}\right|_{r=0}=0$, and the solid black line shows the mean-field boundary [Eq. (4)]. (b) Second derivative of the Wigner distribution at $r=0$ along the line $\kappa_{3}=\kappa_{2} \times 10^{-1.5}$. The point where $\left.W^{\prime \prime}\right|_{r=0}=0$ indicates the disappearance of the local maximum at the origin. (c) Illustrations of the Wigner function corresponding to the points marked in panel (a): by a blue-cross [(c)i], FP state with a single peak in $W(r)$ at the origin; red-cross [(c)ii], bistable state with two peaks in the Wigner function (the contrast $\min \left(W_{0}, W_{+}\right)-W_{-}$is also shown; it is $\approx 10^{-2}$ in this case); and green-cross [(c)iii], limit-cycle state which has a single peak at $r \geqslant 0$.

$r$ [51] $W(r)=\frac{2}{\pi} e^{-2 r^{2}} \sum_{n=0}^{\infty}(-1)^{n} P_{n} L_{n}\left(4 r^{2}\right)$, where $L_{n}$ are the Laguerre polynomials. The sign of the second derivative at $r=0,\left.W^{\prime \prime}(r)\right|_{r=0}=-\frac{8}{\pi} \sum_{n=0}^{\infty}(-1)^{n} P_{n}(1+2 n)$ determines 
whether or not there is a maximum at the origin [see Fig. 7(b)]; hence, when $\left.W^{\prime \prime}(r)\right|_{r=0}>0$ the system is in the LC state. However, this approach leads to a very large bistable region which includes cases where one of the coexisting peaks in the Wigner function can be negligibly small.

To quantify just how small the smaller of the two coexisting peaks in the bistable region is, we defined a contrast function $\min \left(W_{0}, W_{+}\right)-W_{-}$; this compares the smaller of $W_{0}$ and $W_{+}$, which are the values of the Wigner function for the maximas at $r=0$ and $r>0$ respectively, with the value at the local minimum between them, $W_{-}$[see Fig. 7(c)ii]. Figure 7(a) shows this contrast plotted using a logarithmic scaling over the regions where two peaks exist (we note that the Wigner function was found to be positive throughout). The plot shows that while the boundary between the FP and $\mathrm{B}$ regions is pretty sharp, the one between B and LC regions is extremely diffuse. Indeed, the peak at the origin persists over a range of parameters while becoming extremely small, so small that it cannot play a meaningful role in affecting the system's behavior.

Following this argument, it makes sense to set the LC-B boundary at a point where the peak at the origin becomes very small rather than disappearing entirely, though such a choice will always be somewhat arbitrary. For Fig. 2, the LC-B boundary (lower dashed line) to corresponds to a contrast, $\min \left(W_{0}, W_{+}\right)-W_{-}=0.0001$, chosen so that for small $\kappa_{3} / \kappa_{2}$ the bistable region matches up reasonably well with the peak in the second moment of the number distribution.

\section{APPENDIX C: PERTURBATION THEORY}

\section{General method}

Perturbation theory provides a convenient method of calculating the way in which the relative phase distribution behaves for weak coupling. The steady state of the uncoupled $(J=$ 0 ), two-oscillator system [Eq. (7)] only has diagonal terms. Treating the coupling as a perturbation $[20,28]$ allows us to calculate the terms in the first off-diagonal as a function of the uncoupled oscillator terms. Each subsequent off-diagonal can, in turn, be calculated from the previous ones [19].

Writing Eq. (7) in the number state basis, with $\rho_{n, m}^{(p)}=\langle n+$ $p, m|\rho| n, m+p\rangle$, leads to a set of simultaneous equations

$$
\begin{aligned}
\dot{\rho}_{n, m}^{(p)}= & +i J \Delta_{n, m}^{(p)}-\left[G_{n}^{(p)}+G_{m}^{(p)}\right] \rho_{n, m}^{(p)} \\
& +A_{n+1}^{(p)} \rho_{n+1, m}^{(p)}+B_{n-2}^{(p)} \rho_{n-2, m}^{(p)}+C_{n+3}^{(p)} \rho_{n+3, m}^{(p)} \\
& +A_{m+1}^{(p)} \rho_{n, m+1}^{(p)}+B_{m-2}^{(p)} \rho_{n, m-2}^{(p)}+C_{m+3}^{(p)} \rho_{n, m+3}^{(p)},
\end{aligned}
$$

with

$$
\begin{aligned}
\Delta_{n, m}^{(p)}= & -\sqrt{(n+1)(m+p)} \rho_{n+1, m}^{(p-1)}+\sqrt{(m+1)(n+p)} \rho_{n, m+1}^{(p-1)}-\sqrt{n(m+p+1)} \rho_{n-1, m}^{(p+1)}+\sqrt{m(n+p+1)} \rho_{n, m-1}^{(p+1)}, \\
G_{n}^{(p)}= & \frac{1}{2}\left\{\kappa_{1}(2 n+p)+\kappa_{2}[(n+p+1)(n+p+2)+(n+1)(n+2)]\right. \\
& \left.+\kappa_{3}[(n+p)(n+p-1)(n+p-2)+n(n-1)(n-2)]\right\} \\
A_{n+1}^{(p)}= & \kappa_{1} \sqrt{(n+1)(n+p+1)}, \\
B_{n-2}^{(p)}= & \kappa_{2} \sqrt{n(n-1)(n+p)(n+p-1)}, \\
C_{n+3}^{(p)}= & \kappa_{3} \sqrt{(n+1)(n+2)(n+3)(n+p+1)(n+p+2)(n+p+3)} .
\end{aligned}
$$

In the steady state, this reduces to sets of simultaneous equations with the coupling term, $\Delta_{n, m}^{(p)}$ linking together terms with different $p$ values. The zeroth-order terms are the diagonal $(p=0)$ elements, the uncoupled probabilities $\rho_{n, m}^{(0)}=$ $P_{n} P_{m}$, which necessarily sum to unity. The first-order terms are obtained by substituting the zeroth-order terms into the expression for $\Delta_{n, m}^{(p)}$, leading to nonzero contributions for $p=1$ and the process is continued to higher order in $J$ recursively.

The first-order terms obey the relation $\rho_{m, n}^{(1)}=-\rho_{n, m}^{(1)}$ and hence sum to zero [19], which means that they make no contribution to the relative phase distribution [Eq. (9)] since it depends on sums of the off-diagonal elements. The sum of the $p=2$ terms, however, is real and finite and so does contribute resulting in a $\pi$-periodic relative phase distribution. Continuing to higher orders, we find that all of the odd- $p$ terms sum to zero, and so only the even- $p$ sums contribute to the relative phase distribution. In particular, the $p=4$ terms, lead to a $\pi / 2$-periodic contribution which can dominate the phase distribution when the $\pi$-periodic terms vanish.

\section{Low-occupation-number regime}

This calculation can be simplified and solved analytically in the limit of very low photon numbers. We proceed by assuming only the lowest three photon states are appreciably occupied, i.e., $P_{n>2}=0$, and hence truncate the state space to include only $|0\rangle,|1\rangle$, and $|2\rangle$. Because of the size of the Hilbert space, only a single term contributes to the relative phase distribution, $P(\phi)=\frac{1}{2 \pi}+\frac{1}{\pi} \operatorname{Re}\left[e^{2 i \phi} \rho_{0,0}^{(2)}\right]$. In the steady state, Eq. (C1) with $p=2$ leads to

$$
\rho_{0,0}^{(2)}=-i J 2 \sqrt{2}\left(2 \kappa_{1}+14 \kappa_{2}\right)^{-1} \rho_{1,0}^{(1)},
$$

using the relation $\rho_{m, n}^{(1)}=-\rho_{n, m}^{(1)}$. Equation (C1) with $p=1$ gives

$$
\rho_{1,0}^{(1)}=\frac{i J \sqrt{2}\left(P_{1}^{2}-P_{0} P_{2}\right)}{\left(2 \kappa_{1}+13 \kappa_{2}\right)} .
$$

This results in the relative phase distribution

$$
P(\phi)=\frac{1}{2 \pi}+\frac{2 J^{2}\left(P_{1}^{2}-P_{0} P_{2}\right) \cos (2 \phi)}{\pi\left(\kappa_{1}+7 \kappa_{2}\right)\left(2 \kappa_{1}+13 \kappa_{2}\right)} .
$$


This is a $\pi$-periodic distribution with the position of the peaks determined by the steady state of the uncoupled oscillators. In our model $P_{2} P_{0}>P_{1}^{2}$, which leads to peaks at $\pi / 2$ and $3 \pi / 2$.

\section{APPENDIX D: MEAN-FIELD ANALYSIS OF COUPLED OSCILLATORS}

The same mean-field procedure discussed above in Appendix A can be applied to the case of two coupled oscillators with $\alpha=\left\langle\hat{a}_{1}\right\rangle$ and $\beta=\left\langle\hat{a}_{2}\right\rangle$, leading to the equations of motion

$$
\begin{aligned}
& \dot{\alpha}=-\frac{1}{2}\left[\kappa_{1}-2 \kappa_{2}|\alpha|^{2}+3 \kappa_{3}|\alpha|^{4}\right] \alpha-i J \beta, \\
& \dot{\beta}=-\frac{1}{2}\left[\kappa_{1}-2 \kappa_{2}|\beta|^{2}+3 \kappa_{3}|\beta|^{4}\right] \beta-i J \alpha .
\end{aligned}
$$

Changing to polar coordinates, with the definitions $\alpha=r_{1} e^{i \varphi_{1}}$ and $\beta=r_{2} e^{i \varphi_{2}}$, and then rewriting the equations in terms of the sum-and-difference variables $r=r_{1}-r_{2}, R=r_{1}+r_{2}$, and $\phi=\varphi_{1}-\varphi_{2}$, we find

$$
\begin{aligned}
\dot{\phi}= & \frac{4 J r R}{R^{2}-r^{2}} \cos \phi, \\
\dot{r}= & -J R \sin \phi-\frac{\kappa_{1}}{2} r+\frac{\kappa_{2}}{4} r\left(r^{2}+3 R^{2}\right) \\
& -\frac{3 \kappa_{3}}{32} r\left(r^{4}+10 R^{2} r^{2}+5 R^{4}\right) .
\end{aligned}
$$

For large photon occupation numbers (as is the case in the limit-cycle regime) and for weak couplings $\left(J / \kappa_{2} \ll 1\right)$, we have $r \ll R$ and can approximate these equations as

$$
\begin{aligned}
& \dot{\phi} \approx \frac{4 J r}{R} \cos \phi, \\
& \dot{r} \approx-\frac{15 \kappa_{3} R^{4}}{32} r-J R \sin \phi .
\end{aligned}
$$

The weakness of the coupling and the small size of the ratio $r / R$ leads to a separation of timescales with $\phi$ relaxing much more slowly than $r$. Adiabatic elimination of $r$ leads to the simple relation $\dot{\phi}=-\partial U(\phi) / \partial \phi$ with the pseudopotential [44]

$$
U(\phi)=-\frac{16 J^{2}}{15 \kappa_{3} R^{4}} \cos 2 \phi .
$$

This potential predicts relative phase preferences of 0 and $\pi$, its stable minima.

\section{APPENDIX E: PHASE DIFFUSION}

In this Appendix, we return to the case of a single oscillator and obtain an estimate for the phase diffusion rate in the semiclassical limit where photon numbers are large. In the semiclassical regime at least, the strength of phase synchronization in coupled oscillators is determined by competition between the coupling and the rate of phase diffusion in the individual oscillators, with slower phase diffusion leading to stronger phase preferences $[9,19]$.
The phase distribution for a single oscillator takes the form $[19,45,52]$

$$
\begin{aligned}
P(\varphi) & =\frac{1}{2 \pi} \sum_{n, m=0}^{\infty}\langle n|\rho| m\rangle e^{i(m-n) \varphi}, \\
& =\frac{1}{2 \pi}+\frac{1}{\pi} \operatorname{Re}\left[\sum_{k=1}^{\infty} e^{i k \varphi} \Phi^{(k)}\right],
\end{aligned}
$$

with $\Phi^{(k)}=\sum_{n=0}^{\infty} \rho_{n}^{(k)}$, where $\rho_{n}^{(k)}=\langle n|\rho| n+k\rangle$. Although the behavior is in general quite complex, we can obtain a simple approximate description in the semiclassical limit where the density matrix is tightly peaked around a large average photon occupation number $[19,36]$.

Using Eq. (1) [and the notation introduced in (C1)], we can obtain the equation of motion for $\Phi^{(k)}$ :

$$
\dot{\Phi}^{(k)}=\sum_{n}\left[-G_{n}^{(k)}+A_{n}^{(k)}+B_{n}^{(k)}+C_{n}^{(k)}\right] \rho_{n}^{(k)} .
$$

In the semiclassical limit, i.e., the strong gain regime where $\gamma=\kappa_{1} / \kappa_{2} \ll 1$ and $\Gamma=\kappa_{3} / \kappa_{2} \ll 1$, the photon number saturates to a large value $\langle n\rangle \simeq 2 \kappa_{2} /\left(3 \kappa_{3}\right)$ [see Eq. (A4)]. We proceed by assuming we can replace $n$ by $\langle n\rangle$ and expand the square roots appearing in $A_{n}^{(k)}, B_{n}^{(k)}$, etc., treating $1 /\langle n\rangle$ together with $\gamma$ and $\Gamma$ as small quantities [19,36,53]. This leads to the simplified equation

$$
\dot{\Phi}^{(k)}=\kappa_{2}\left[-\frac{5}{4} k^{2}+\mathcal{O}\left(\gamma, \Gamma,\langle n\rangle^{-1}\right)\right] \Phi^{(k)} .
$$

Hence, to leading order, the relaxation timescale for the $k$ th component, $\Phi^{(k)}$, is simply proportional to $1 / \kappa_{2}, \tau_{k}^{L C} \kappa_{2} \simeq$ $4 /\left(5 k^{2}\right)$.

Notice that the semiclassical approximation here assumes all the off-diagonal elements $\left(\rho_{n}^{(k)}\right)$ decay at the same rate, and hence the decay time for $k=1$ is also an approximate linewidth for the oscillator [36]. The slowest timescales $\tau_{k}$ associated with the matrices $\mathcal{M}^{(k)}$ in Eq. (2) are found to plateau in the limit of $\kappa_{1} / \kappa_{2} \ll 1$ and $\kappa_{3} / \kappa_{2} \ll 1$ [illustrated on the left-hand side of Fig. 3(a)] and numerically we find $\tau_{1} \simeq 0.8 / \kappa_{2}$ and $\tau_{2} \simeq 0.2 / \kappa_{2}$ in this regime, matching up very well with $\tau_{1}^{L C}$ and $\tau_{2}^{L C}$, respectively.

Finally, using the definition Eqs. (E2) and (E4), we see that within this approximation the phase distribution obeys a diffusion equation $[19,53]$ :

$$
\dot{P}(\varphi)=\frac{5 \kappa_{2}}{4} \frac{\partial^{2} P(\varphi)}{\partial \varphi^{2}} .
$$

This is very different from what is obtained in a similar calculation for the QvdP oscillator (or indeed the laser [36]), where the diffusion constant is $\propto 1 /\langle n\rangle$. Hence, for such systems phase diffusion gets weaker (and the linewidth narrower), so that synchronization effects get stronger, as the photon number increases [16].
[1] M. Dykman, Fluctuating Nonlinear Oscillators (Oxford University Press, Oxford, UK, 2012).
[2] F. R. Ong, M. Boissonneault, F. Mallet, A. PalaciosLaloy, A. Dewes, A. C. Doherty, A. Blais, P. Bertet, 
D. Vion, and D. Esteve, Phys. Rev. Lett. 106, 167002 (2011).

[3] F. R. Ong, M. Boissonneault, F. Mallet, A. C. Doherty, A. Blais, D. Vion, D. Esteve, and P. Bertet, Phys. Rev. Lett. 110, 047001 (2013).

[4] Z. Wang, M. Pechal, E. A. Wollack, P. Arrangoiz-Arriola, M. Gao, N. R. Lee, and A. H. Safavi-Naeini, Phys. Rev. X 9, 021049 (2019).

[5] Y. Zhang, J. Gosner, S. M. Girvin, J. Ankerhold, and M. I. Dykman, Phys. Rev. A 96, 052124 (2017).

[6] S. Puri, C. K. Andersen, A. L. Grimsmo, and A. Blais, Nat. Comms. 8, 15785 (2017).

[7] M. I. Dykman, C. Bruder, N. Lörch, and Y. Zhang, Phys. Rev. B 98, 195444 (2018).

[8] R. Rota, F. Minganti, C. Ciuti, and V. Savona, Phys. Rev. Lett. 122, 110405 (2019).

[9] A. Pikovsky, M. Rosenblum, and J. Kurths, Synchronisation: A Universal Concept in Nonlinear Sciences (Cambridge University Press, Cambridge, UK, 2001).

[10] J. A. Acebrón, L. L. Bonilla, C. J. Vicente, F. Ritort, and R. Spigler, Rev. Mod. Phys. 77, 137 (2005).

[11] J. D. Cresser, W. H. Louisell, P. Meystre, W. Schleich, and M. O. Scully, Phys. Rev. A 25, 2214 (1982).

[12] O. V. Zhirov and D. L. Shepelyansky, Eur. Phys. J. D 38, 375 (2006).

[13] G. Manzano, F. Galve, G. L. Giorgi, E. Hernández-García, and R. Zambrini, Sci. Rep. 3, 1439 (2013).

[14] M. Ludwig and F. Marquardt, Phys. Rev. Lett. 111, 073603 (2013).

[15] A. Mari, A. Farace, N. Didier, V. Giovannetti, and R. Fazio, Phys. Rev. Lett. 111, 103605 (2013).

[16] T. E. Lee and H. R. Sadeghpour, Phys. Rev. Lett. 111, 234101 (2013).

[17] S. Walter, A. Nunnenkamp, and C. Bruder, Phys. Rev. Lett. 112, 094102 (2014).

[18] N. Lörch, E. Amitai, A. Nunnenkamp, and C. Bruder, Phys. Rev. Lett. 117, 073601 (2016).

[19] C. Davis-Tilley and A. D. Armour, Phys. Rev. A 94, 063819 (2016).

[20] N. Lörch, S. E. Nigg, A. Nunnenkamp, R. P. Tiwari, and C. Bruder, Phys. Rev. Lett. 118, 243602 (2017).

[21] V. Ameri, M. Eghbali-Arani, A. Mari, A. Farace, F. Kheirandish, V. Giovannetti, and R. Fazio, Phys. Rev. A 91, 012301 (2015).

[22] M. R. Hush, W. Li, S. Genway, I. Lesanovsky, and A. D. Armour, Phys. Rev. A 91, 061401(R) (2015).

[23] T. E. Lee, C.-K. Chan, and S. Wang, Phys. Rev. E 89, 022913 (2014).

[24] W. Li, C. Li, and H. Song, Phys. Rev. E 93, 062221 (2016).

[25] F. Galve, G. Luca Giorgi, and R. Zambrini, Quantum correlations and synchronization measures, in Lectures on General
Quantum Correlations and their Applications, edited by F. F. Fanchini, D. d. O. Soares Pinto, and G. Adesso (Springer International, Cham, 2017), pp. 393-420.

[26] A. Roulet and C. Bruder, Phys. Rev. Lett. 121, 053601 (2018).

[27] A. Roulet and C. Bruder, Phys. Rev. Lett. 121, 063601 (2018).

[28] M. Koppenhöfer and A. Roulet, Phys. Rev. A 99, 043804 (2019).

[29] M. Xu, D. A. Tieri, E. C. Fine, J. K. Thompson, and M. J. Holland, Phys. Rev. Lett. 113, 154101 (2014).

[30] B. Zhu, J. Schachenmayer, M. Xu, F. Herrera, J. G. Restrepo, M. J. Holland, and A. M. Rey, New J. Phys. 17, 083063 (2015).

[31] T. Weiss, A. Kronwald, and F. Marquardt, New J. Phys. 18, 013043 (2016).

[32] S. E. Nigg, Phys. Rev. A 97, 013811 (2018).

[33] O. V. Zhirov and D. L. Shepelyansky, Phys. Rev. Lett. 100, 014101 (2008).

[34] G. Lindblad, Commun. Math. Phys. 48, 119 (1976).

[35] C. W. Gardiner and P. Zoller, Quantum Noise (Springer, Berlin, 2000).

[36] M. O. Scully and M. S. Zubairy, Quantum Optics (Cambridge University Press, Cambridge, UK, 1997).

[37] O. Scarlatella, A. A. Clerk, and M. Schirò, New J. Phys. 21, 043040 (2019).

[38] D. A. Rodrigues, J. Imbers, and A. D. Armour, Phys. Rev. Lett. 98, 067204 (2007).

[39] J. R. Nielsen and C. Kittel, Am. Math. Mon. 66, 928 (2006).

[40] H. Risken, C. Savage, F. Haake, and D. F. Walls, Phys. Rev. A 35, 1729 (1987).

[41] M. Nirmal, B. O. Dabbousi, M. G. Bawendi, J. J. Macklin, J. K. Trautman, T. D. Harris, and L. E. Brus, Nature (London) 383, 802 (1996).

[42] K. Macieszczak, M. Guţă, I. Lesanovsky, and J. P. Garrahan, Phys. Rev. Lett. 116, 240404 (2016).

[43] M. B. Plenio and P. L. Knight, Rev. Mod. Phys. 70, 101 (1998).

[44] A. P. Kuznetsov, N. V. Stankevich, and L. V. Turukina, Phys. D Nonlinear Phenom. 238, 1203 (2009).

[45] S. M. Barnett and D. T. Pegg, Phys. Rev. A 42, 6713 (1990).

[46] A. Luis and L. L. Sánchez-Soto, Phys. Rev. A 53, 495 (1996).

[47] R. Barak and Y. Ben-Aryeh, J. Opt. B Quantum Semiclassical Opt. 7, 123 (2005).

[48] K. Ishibashi and R. Kanamoto, Phys. Rev. E 96, 052210 (2017).

[49] C. Davis-Tilley, C. K. Teoh, and A. D. Armour, New J. Phys. 20, 113002 (2018).

[50] N. Lörch, Y. Zhang, C. Bruder, and M. I. Dykman, Phys. Rev. Res. 1, 023023 (2019).

[51] L. Gilles, B. M. Garraway, and P. L. Knight, Phys. Rev. A 49, 2785 (1994).

[52] P. L. Knight and C. C. Gerry, Introductory Quantum Optics (Cambridge University Press, Cambridge, UK, 2004).

[53] W. C. Schieve and R. R. McGowan, Phys. Rev. A 48, 2315 (1993). 